Trauma Berufskrankh 2010 - 12[Suppl 2]:132-141 DOI 10.1007/s10039-010-1611-1

Online publiziert: 9. April 2010

(c) Springer-Verlag 2010

\author{
W. Strecker ${ }^{1} \cdot$ G. Suger ${ }^{2} \cdot$ M. Schulte ${ }^{3}$ \\ ${ }^{1}$ Klinik für Orthopädie und Unfallchirurgie, Klinikum am Bruderwald, Bamberg \\ ${ }^{2} \mathrm{Ulm}$ \\ ${ }^{3}$ Klinik für Unfall- und Wiederherstellungschirurgie, \\ Orthopädische Chirurgie, Diakoniekrankenhaus Rotenburg, Rotenburg (Wümme)
}

\section{Das destruierte Hüftgelenk}

\section{Arthrodese vs. Endoprothese}

Der alloarthroplastische Hüftgelenkersatz zählt zu den häufigsten und gleichzeitig erfolgreichsten operativen Interventionen in der Orthopädie und Unfallchirurgie. Die Implantation von Hüftendoprothesen ist derzeit als Goldstandard in der Behandlung der fortgeschrittenen Koxarthrose unterschiedlicher Ätiologie zu betrachten. Dennoch hat auch diese Operationsmethode ihre spezifischen Grenzen, sodass im Einzelfall die Frage nach adäquaten Behandlungsalternativen im Raum steht. Grundsätzlich ist bei geeigneten Indikationen, insbesondere bei jüngeren Patienten, der Stellenwert der Hüftarthrodese zu prüfen. Andere Konstellationen lassen weder eine Alloarthroplastik noch eine Hüftarthrodese zu. Daher sind weitere differenzialtherapeutische Optionen zu berücksichtigen, wie Girdlestone-Anlage, Hüftexartikulation oder "pelvic support osteotomy“.

\section{Hüftarthrodese}

Die operative Versteifung des Hüftgelenks bewirkt eine dauerhafte lokale Schmerzfreiheit bei mechanisch hoher Belastbarkeit. Bei intakten Nachbargelenken einschließlich des kontralateralen Hüftgelenks, beider Kniegelenke und der Lendenwirbelsäule kann für viele Jahre eine gute funktionelle Adaptation erwartet werden [1]. Nach einem Zeitraum von etwa 15-20 Jahren treten allerdings Rückenbeschwerden in bis zu 50-60\% der Patienten oder Kniebeschwerden auf $[1,8]$.
Hierbei werden Gonalgien in einer Häufigkeit von $30-76 \%$ auf der betroffenen und von $13-33 \%$ auf der kontralateralen Seite angegeben.

In Anbetracht der überzeugenden Vorteile der Hüftendoprothetik wird eine primäre Hüftgelenkversteifung von Patienten heutzutage kaum mehr akzeptiert. Aus dieser Entwicklung und der aktuellen Studienlage lassen sich entsprechende Indikationen und Kontraindikationen ableiten.

\section{Indikationen}

Hier sind anzuführen:

- Verlust der hüftstabilisierenden Muskulatur

- Posttraumatische Funktionseinschränkungen, insbesondere bei ausgeprägten heterotopen Ossifikationen

- Hüftkopfnekrosen

- Septische Koxarthritis

- Latent hohes Infektrisiko, insbesondere im tropischen Umfeld

Immunkompromittierende Pathologien, wie Sichelzellenerkrankung, HIV-Seropositivität (HIV: „,human immunodeficiency virus") usw. sowie limitierte regionale infrastrukturelle Voraussetzungen können die Indikation zum alloarthroplastischen Hüftgelenkersatz relativieren.

Hüftarthrodesen können bei jungen und körperlich aktiven Patienten mit den oben genannten Pathologien erwogen werden. Hierbei sollte die Körpergrö- ße $170-175 \mathrm{~cm}$ nicht überschreiten. Die Beweglichkeit in der Lendenwirbelsäule, dem kontralateralen Hüftgelenk und in beiden Kniegelenken sollte unbedingt gegeben sein [10].

\section{Kontraindikationen}

Als absolute Kontraindikation gelten Funktionseinschränkungen der oben genannten benachbarten Gelenke.

Relative Kontraindikationen bestehen bei Patienten mit einer Körpergröße von über $175 \mathrm{~cm}$. Frauen sind ebenso von einer Hüftarthrodese zu verschonen wie Personen mit überwiegend sitzender Tätigkeit [10].

\section{Operationstechnik}

Kreuzplatte. Über einen lateralen $\mathrm{Zu}$ gang erfolgt in Rückenlage nach Luxation des Hüftkopfes die Entknorpelung der Gelenkflächen. Nach Trochanterosteotomie wird die Kreuzplatte, auch CobraPlatte genannt, auf der Außenfläche des Os ileum und der Lateralseite des Femurs fixiert [9].

Ventrale Platte. Über einen anterioren Zugang (Smith-Patterson-Zugang) wird das Gelenk, ggf. unter Zuhilfenahme eines Distraktors, entknorpelt. Nach endgültiger Einstellung erfolgt die Retention durch eine ventral aufgelegte breite DC-Platte (DC: dynamische Kompressionsplatte), proximal der Linea termina- 
lis aufliegend, über die Eminentia iliopectinea zur Vorderseite des proximalen $\mathrm{Fe}$ murs ziehend [7].

\section{Zielgeometrie}

Folgende Hüftgeometrie ist unter Berücksichtigung der beruflichen Beanspruchung mit jedem einzelnen Patienten individuell anzustreben:

- Flexion: $10-25^{\circ}$

- Abduktion/Adduktion: neutral

- Beinverkürzung um 1,0-1,5 cm

- Neutrale rotatorische Einstellung

In der Literatur wurden jedoch auch gewisse Abweichungen von dieser Zielgeometrie angegeben. Die Toleranzen hierbei reichen von $4-7^{\circ}$ Adduktion, $5^{\circ}$ Abduktion sowie bis $\mathrm{zu} 5^{\circ}$ außenrotatorische Einstellung $[3,10]$.

\section{Ergebnisse}

Beide Arthrodesetechniken erreichen in rund 90\% der Fälle ohne eine weitere Revisionsoperation eine primäre knöcherne Durchbauung $[7,9]$. Andere Autoren gehen von $10-20 \%$ Pseudarthrosen sowie 419\% Femurfrakturen im weiteren Verlauf aus [10].

Spätestens 15-20 Jahre nach der Hüftarthrodese ist mit Beschwerden zu rechnen [10]:

- im Bereich der Wirbelsäule in einer Häufigkeit von $50-78 \%$,

- mit einer ipsilateralen Gonalgie in $30-76 \%$ der Fälle,

- mit einer kontralateralen Gonalgie bei $13-33 \%$ der Patienten und

- mit einer kontralateralen Koxalgie in $13-32 \%$ der Fälle.

\section{Hüftendoprothese}

Während bei älteren Patienten mit Koxarthrose unterschiedlicher Ätiologie der alloarthroplastische Hüftgelenkersatz überwiegend gute und sehr gute klinische und funktionelle Ergebnisse aufweist, ist die Operationsindikation für diese Technik bei jüngeren Patienten durchaus kritisch zu hinterfragen.

Eine Analyse von 218 Totalendoprothesen bei 141 Patienten, die zum Operationszeitpunkt 40 Jahre und jünger waren,

Trauma Berufskrankh 2010 - 12[Suppl 2]:132-141 DOI 10.1007/s10039-010-1611-1

(c) Springer-Verlag 2010

\section{W. Strecker · G. Suger · M. Schulte Das destruierte Hüftgelenk. Arthrodese vs. Endoprothese}

\section{Zusammenfassung}

Aufgrund großer Fortschritte im alloarthroplastischen Hüftgelenkersatz verbleiben nur noch wenige Indikationen für die Hüftarthrodese, etwa beim völligen Verlust der hüftstabilisierenden Muskulatur. Die endoprothetischen Möglichkeiten beschränken sich nicht nur auf allgemein bekannte Standardverfahren, sondern müssen individuell maßgeschneiderte Tumorprothesen unter Einschluss des Beckenteilersatzes ebenso berücksichtigen, wie Rückzugsmöglichkeiten mittels Sattelprothese, Kombinationen von Alloarthroplastik und Korrekturosteotomie. Eine funktionell überzeugende Alternative gerade für jüngere Patienten nach frühkindlicher bakterieller Koxitis mit entspre- chendem Verlust von Hüftkopf und Schenkelhals stellt die "pelvic support osteotomy" dar. Darüber hinaus gehende Rückzugsmöglichkeiten bestehen in einer Girdlestone-Anlage und letztlich in einer Hüftexartikulation. Nach Hüftarthrodese mit entsprechenden konsekutiven Beschwerden der Nachbargelenke ist im Einzelfall die Indikation zur reorientierenden Desarthrodese zu prüfen. Für alle genannten Verfahren und Operationstechniken werden entsprechende Beispiele vorgestellt.

\section{Schlüsselwörter}

Alloarthroplastischer Hüftgelenkersatz . "pelvic support osteotomy" · GirdlestoneAnlage $\cdot$ Hüftexartikulation $\cdot$ Hüftarthrodese

\section{Hip joint destruction. Arthrodesis vs endoprosthesis}

\section{Abstract}

Because of the considerable progress achieved in alloplastic hip joint replacement, very few indications for arthrodesis remain, for instance loss of the stabilizing muscles in the hip. The possibilities for total hip arthroplasty are not only limited to the generally accepted standard procedures, but should also take individually customized tumor prostheses into consideration including partial pelvis replacement as well as fallback options using a saddle prosthesis and combinations of alloplastic implants and corrective osteotomy. Pelvic support osteotomy represents a convincing functional alternative particularly for younger patients after bacterial coxitis in early childhood with corresponding loss of the femoral head and neck. Further options consist of Girdlestone resection arthroplasty and ultimately hip disarticulation. After hip arthroplasty accompanied by subsequent complaints in the neighboring joints the indication for reorienting de-arthrodesis should be assessed on an individual basis. Relevant examples are presented for all of the procedures and surgical techniques mentioned.

\section{Keywords}

Alloplastic hip replacement - Pelvic support osteotomy - Girdlestone resection arthroplasty $\cdot$ Hip disarticulation $\cdot$ Hip arthrodesis 


\section{Arthrodese vs. Gelenkersatz}
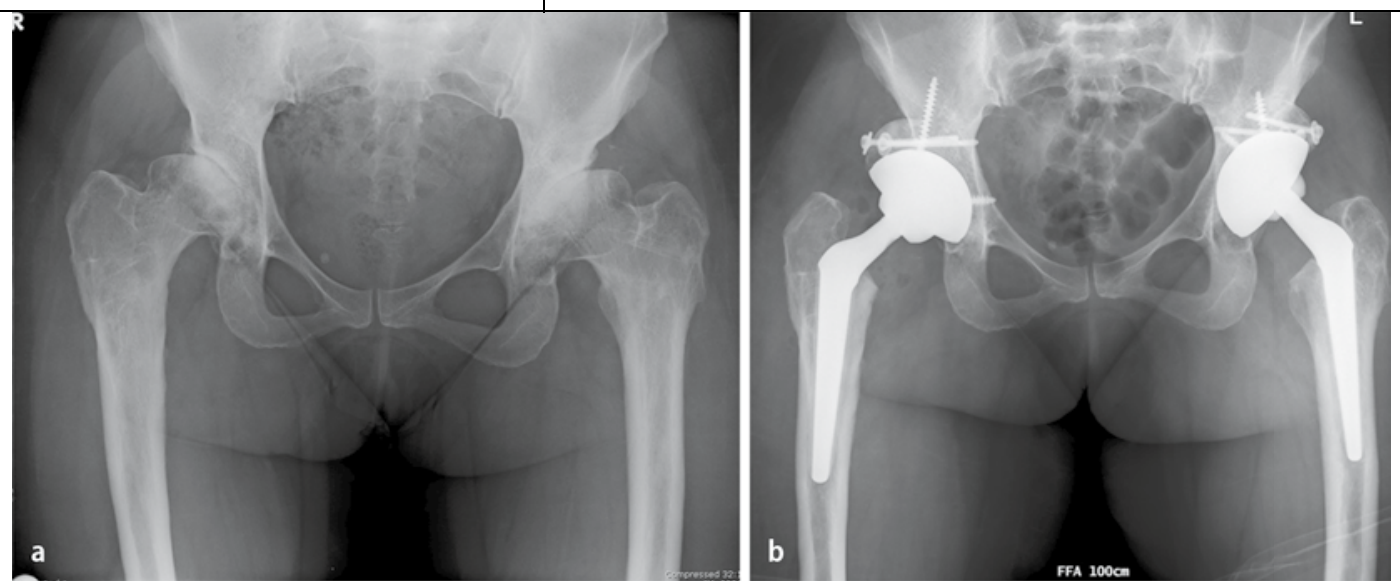

Abb. 1 443-jährige Patientin mit fortgeschrittener Dysplasiekoxarthrose beidseits und Zustand nach bilateraler intertrochanterer Varisationsaußentorsionsosteotomie (a), Zustand nach beidseitiger autogener Pfannendachplastik und Implantation einer zementfreien Hüfttotalendoprothese mit torsionsneutralem Konusschaft (b)
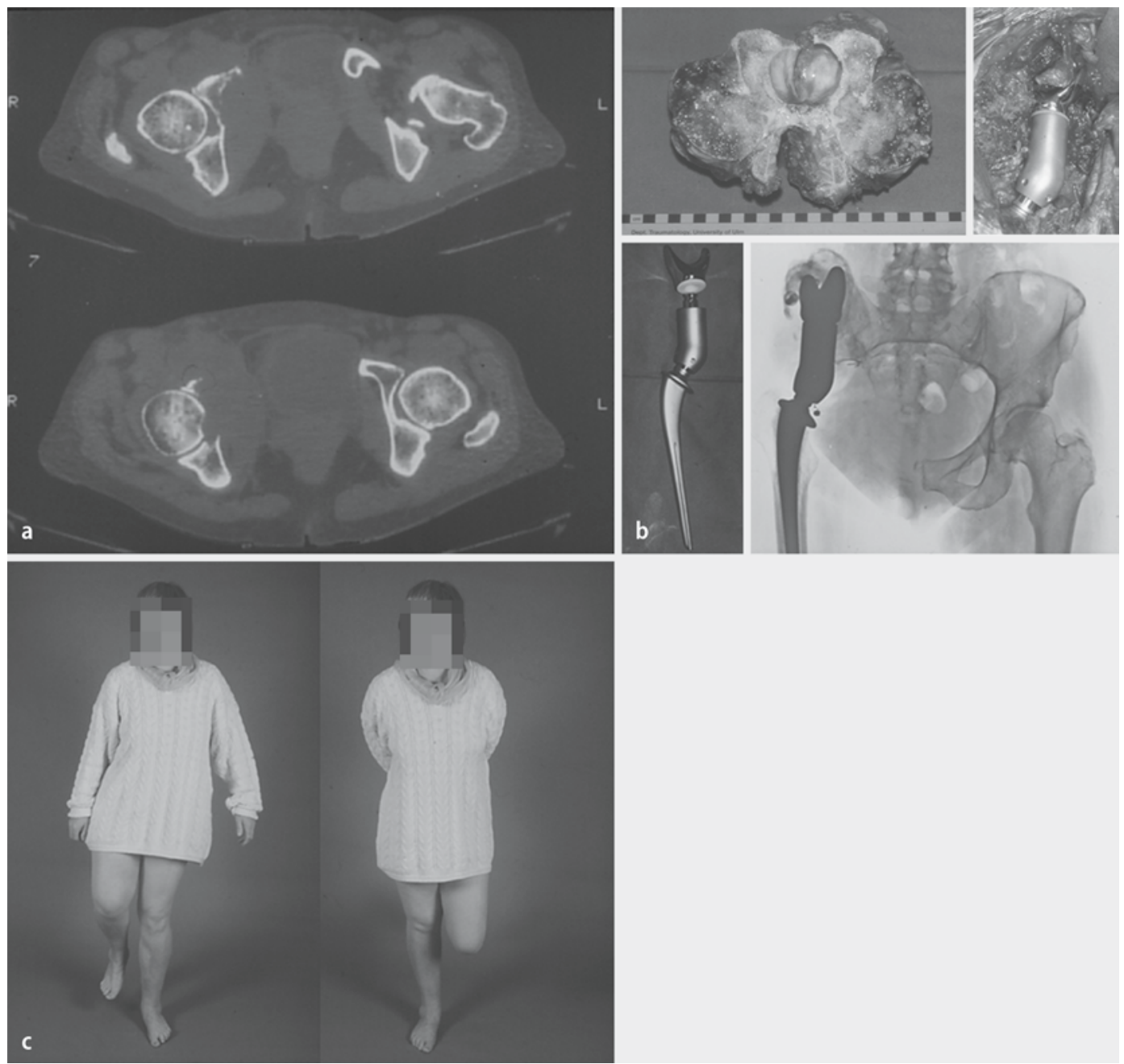

Abb. 2 477-jährige Patientin mit malignem fibrösem Histiozytom azetabulär rechts, a Computertomographie, b Implantation einer Sattelprothese nach R0-Resektion, c postoperativ gute Belastbarkeit bei allerdings eingeschränkter Hüftgelenkbeweglichkeit

zeigte nach 20 Jahren eine Überlebensrate von $84 \%$ für die Pfannenkomponente, von $86 \%$ für die Schaftkomponente und für beide zusammen von $75 \%$. Besonders ungünstig waren die Ergebnisse bei jungen Männern, bei welchen die 20-JahresÜberlebensrate lediglich bei $55 \%$ lag, sowie für Patienten mit primärer Koxarthrose, bei denen nach 20 Jahren mehr als 50\% der Hüftendoprothesen bereits revidiert oder gewechselt worden waren [2].

Inwieweit eine zementfreie Verankerungstechnik der Prothesenkomponenten bessere Resultate bei jüngeren Patienten erbringen wird, muss die Zukunft zeigen. Bis jetzt fehlen diesbezüglich valide publizierte Langzeitergebnisse.

\section{Endoprothesenformen}

Die unterschiedlichen Formen von zementfreien (• Abb. 1) und zementierten Hüftendoprothesen, einschließlich der Hybridvarianten, gehören zum Allgemeingut der orthopädischen Chirurgie und müssen daher hier nicht vorgestellt werden. Weniger bekannt und daher von größerem Interesse sind Sonderformen 
der Hüftendoprothetik, die in Einzelfällen als Rettungsanker dienen können.

\section{Sattelprothese}

Ausgedehnte azetabuläre Knochendefekte, sei es postinfektiöser oder posttraumatischer Genese oder nach radikaler Tumorresektion, stellen eine hohe Herausforderung bezüglich ihrer anatomischen Rekonstruktion und funktionellen Wiederherstellung dar. Bei adäquater knöcherner Abstützung am Os ileum kranial kann die Implantation einer Sattelprothese im Einzelfall eine sinnvolle und operativ relativ schonende Maßnahme darstellen [4]. Nachteilig ist, bedingt durch die erheblich veränderte Hüftgeometrie, eine deutliche funktionelle Bewegungseinschränkung (• Abb. 2).

Bei Tumorpatienten ist bei einem adäquaten rezidivfreien Intervall eine sekundäre Konversion in eine Tumorendoprothese im Sinne eines Beckenteilersatzes zu erwägen.

\section{Beckenteilersatz}

Bei hochgradigen Azetabulumdefekten, sei es posttraumatisch oder nach kurativer Malignomresektion, und quoad vitam guter Langzeitprognose sind die maßgeschneiderte Tumorendoprothese - derartige Sonderimplantate werden auf der Grundlage eines CT-Datensatzes (CT: Computertomographie) individuell angefertigt - bzw. der Beckenteilersatz die Therapie der Wahl. Systemisch und lokal ausreichende Biologie, insbesondere auch eine adäquate Qualität des zur Verankerung zur Verfügung stehenden Restbeckens, sowie ein motivierter und kooperativer Patient sind hierfür unabdingbare Voraussetzungen (• Abb. 3).

\section{Sonderfälle}

Eine Reihe genetischer, metabolischer oder anatomischer Konstellationen schließt eine Arthrodese des Hüftgelenks aus. Andererseits entziehen sich derartige Situationen auch jeder standardisierten Hüftalloarthroplastik. Im Folgenden seien nur einige Beispiele angeführt.
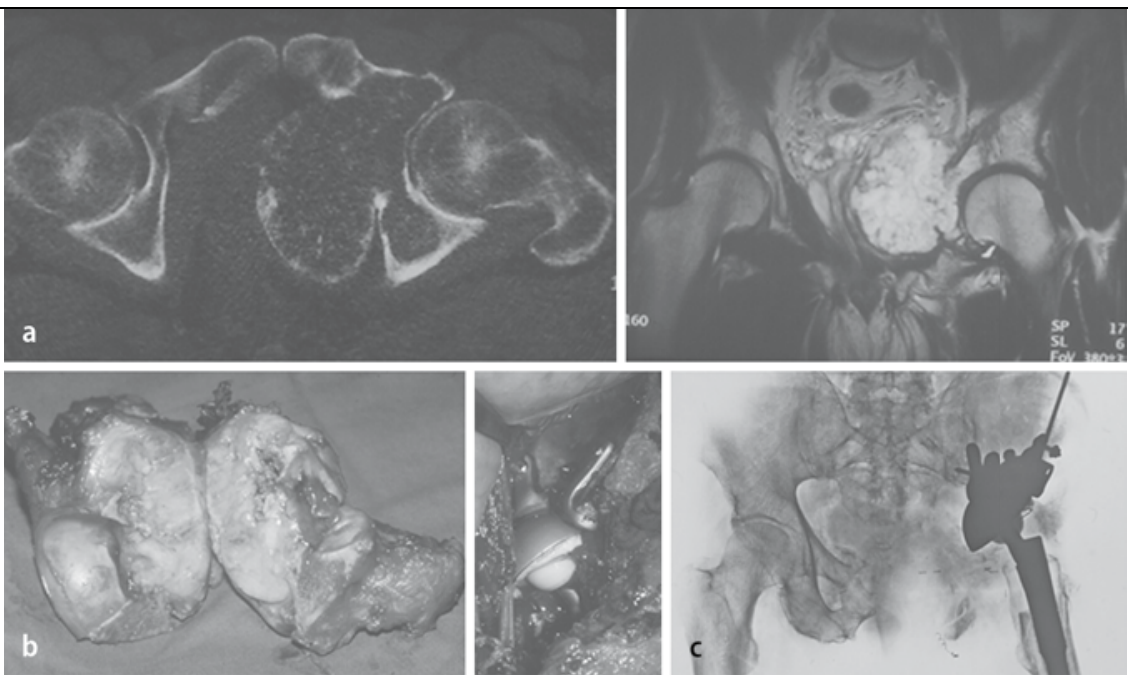

Abb. $3 \Delta$ 62-jähriger Patient mit Chondrosarkom G1 azetabulär links mit deutlicher mechanischer Verdrängung der anatomischen Nachbarstrukturen im kleinen Becken (a), nach Beckenteilresektion vom Typ Ila nach Enneking Implantation eines sich kranial am linken Os ileum abstützenden Beckenteilersatzes (b), Vervollständigung der Alloarthroplastik durch Einzementieren einer Polyethylenpfanne und Verwendung eines modularen Revisionsschaftes mit Keramikkopf (c)
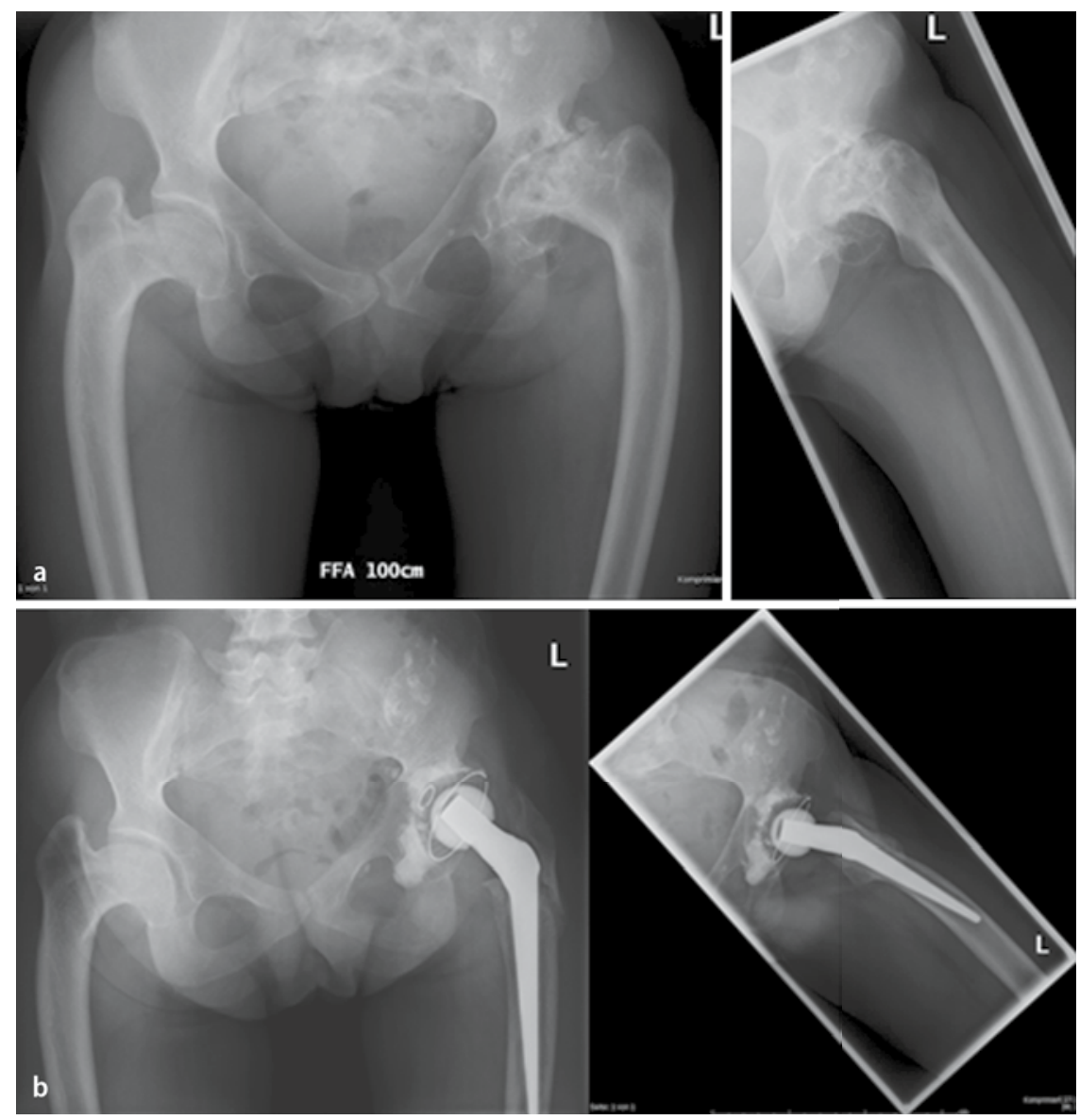

Abb. $4 \Delta$ Ossäre Dystrophie bei renalem Minderwuchs, a hochgradige Destruktion des linken Hüftgelenks mit Varusdeformität des proximalen Femurs, b zementierte Implantation einer Polyethylenpfanne sowie zementfreies Einsetzen eines sonderangefertigten Prothesenschaftes 


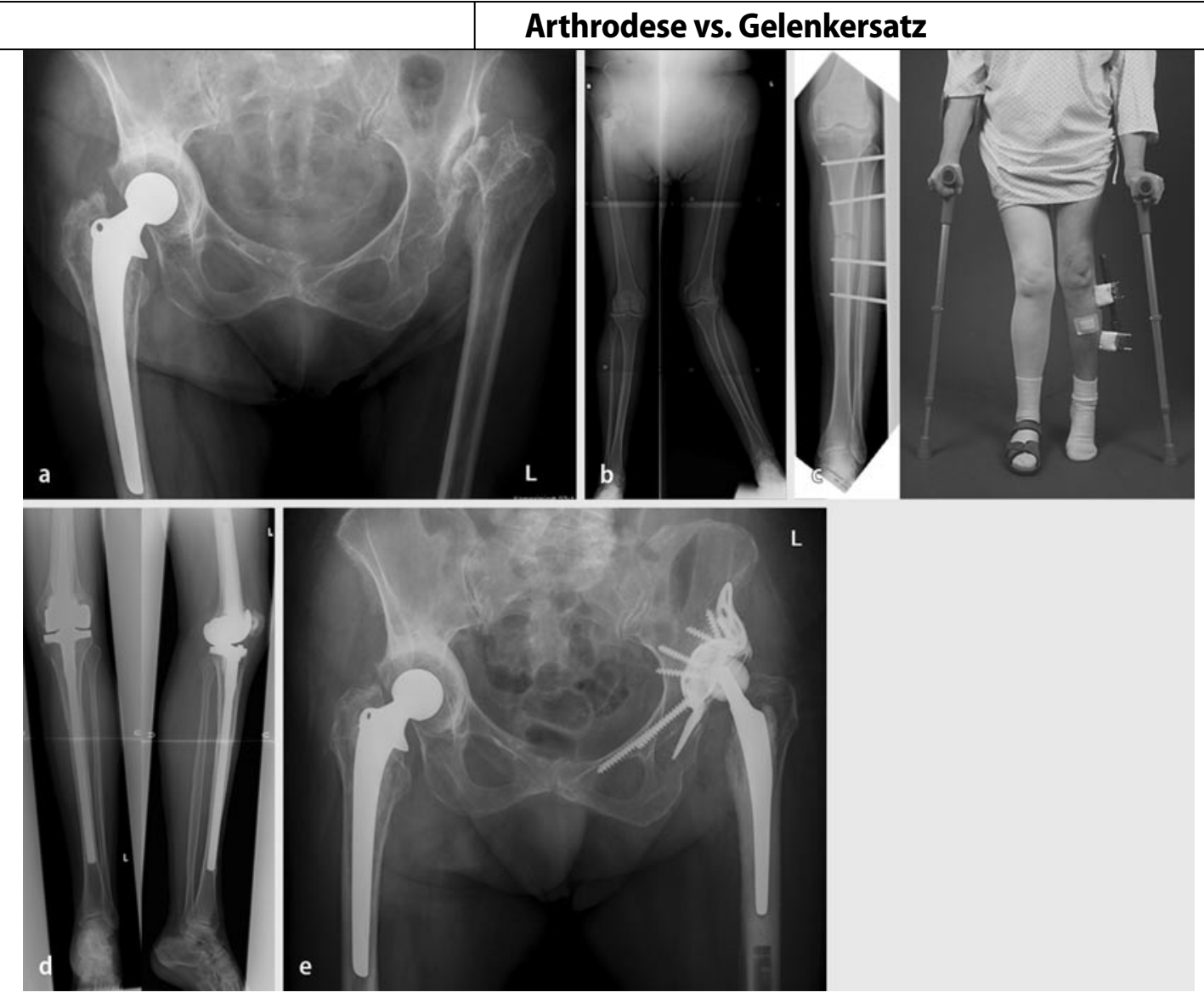

\begin{abstract}
Abb. 5 < Kongenitale Hypoplasie des linken Hüftgelenks (a) mit gleichzeitig kapsulo-ligamentär instabiler Valgusgonarthrose IV ipsilateral (b), kontinuierliche diaphysäre Varisation des linken Unterschenkels mittels unilateralem Distraktionsfixateur (c), Implantation einer gekoppelten trikompartimentellen Knieendoprothese mit langem tibialem Stiel (d), Rekonstruktion des linken Hüftgelenks (e)
\end{abstract}

\section{Ossäre Dystrophie bei renalem Minderwuchs}

Beispielhaft sei der Fall einer 38-jährigen Patientin mit renalem Minderwuchs $(123 \mathrm{~cm} / 25 \mathrm{~kg}$ ), Zustand nach 2-maliger Nierentransplantation und dementsprechend langjähriger Immunsuppression angeführt. Zudem lagen Analgetika- und Nikotinabusus vor.

Es bestand eine hochgradige Destruktion des linken Hüftgelenks mit Varusdeformität des proximalen Femurs (• Abb. 4a). Nach ausführlicher dreidimensionaler Analyse wurden eine Polyethylenpfanne zementiert implantiert sowie ein sonderangefertigter Prothesenschaft zementfrei eingesetzt (- Abb. 4b).

\section{Kongenitale Hypoplasie des linken Hüftgelenks}

In - Abb. 5 ist der Fall einer 74-jährigen Patientin mit kongenitaler Hypoplasie des linken Hüftgelenks mit gleichzeitig kapsulo-ligamentär instabiler Valgusgonarthrose Grad IV ipsilateral vorgestellt.

Zunächst erfolgte eine kontinuierliche diaphysäre Varisation des linken Unter- schenkels mittels unilateralem Distraktionsfixateur zur Vermeidung von neurovaskulären und muskulären Dehnungsschäden. Nach erreichter tibialer Achsausgradung wurde eine gekoppelte trikompartimentelle Knieendoprothese mit langem tibialem Stiel implantiert und erst im letzten Schritt das linke Hüftgelenk durch autogene Pfannenbodenplastik, Einpassen eines Burch-Schneider-Ringes und Implantation einer zementierten HüftTEP (TEP: Totalendoprothese) mit Polyethylen-Keramik-Gleitpaarung rekonstruiert.

\section{Koxofemorale Destruktion nach Tumorresektion und Radiochemotherapie}

Bei der 22-jährigen Patientin waren im 4. Lebensjahr eine Neuroblastommetastase im rechten Schenkelhals reseziert (- Abb. 6a) und nachfolgend eine Chemotherapie durchgeführt worden. Im 7. Lebensjahr erfolgte eine intertrochantere Valgisationsosteotomie. Die daraus resultierende Destruktion des rechten Hüftgelenks mit hochgradig verkürztem und valgisiertem Schenkelhals sowie die Verkürzung des rechten Beines um $6,5 \mathrm{~cm}$ wurden durch autogene Pfannendachplastik, subtrochantere Varisationsosteotomie und Implantation einer zementfreien Hüft-TEP mit Keramik-Keramik-Gleitpaarung und torsionsneutralem Konusschaft versorgt (• Abb. 6b). Trotz Neurolyse des N. ischiadicus ließen sich bei vorbestehenden Vernarbungen lediglich $3,5 \mathrm{~cm}$ an Länge gewinnen. Daher erfolgte zusätzlich eine treppenförmige einzeitige Verlängerungsosteotomie des distalen Femurs um 2,5 cm (• Abb. 6c).

\section{Posttraumatische Osteodystrophie}

Die 50-jährige Patientin hatte nach einer Schenkelhalsfraktur im Kindesalter eine posttraumatische Osteodystrophie des linken Hüftgelenks und des linken Beins entwickelt, woraus ein proximaler Femurdefekt mit $11 \mathrm{~cm}$ Verkürzung resultierte (- Abb. 7a). Nach ausführlicher räumlicher Analyse der knöchernen Geometrie und klinischer Überprüfung der Muskelfunktion, die durch MRT (Magnetresonanztomographie) verifiziert wurde, wurde gemeinsam mit der Patientin der Entschluss zur alloarthroplastischen Rekon- 

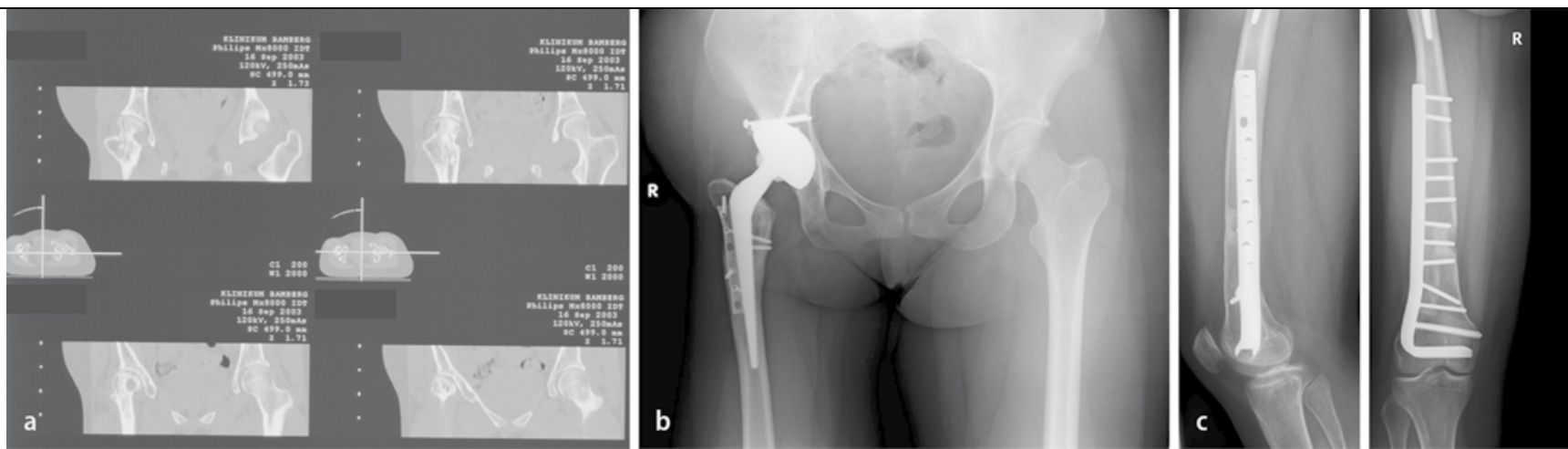

Abb. $6 \Delta$ Koxofemorale Destruktion nach Tumorresektion und Radiochemotherapie, a Resektion einer Neuroblastommetastase im rechten Schenkelhals im 4. Lebensjahr, b autogene Pfannendachplastik, subtrochantere Varisationsosteotomie und Implantation einer zementfreien Hüft-TEP mit Keramik-Keramik-Gleitpaarung und torsionsneutralem Konusschaft, $\mathbf{c}$ treppenförmige Verlängerungsosteotomie des distalen Femurs

Abb. 7 Posttrauma-

tische Osteodystrophie und proximaler Femurdefekt mit $11 \mathrm{~cm}$ Verkürzung

(a), alloarthroplastische Rekonstruktion unter Verwendung eines Spezialschaftes (b)
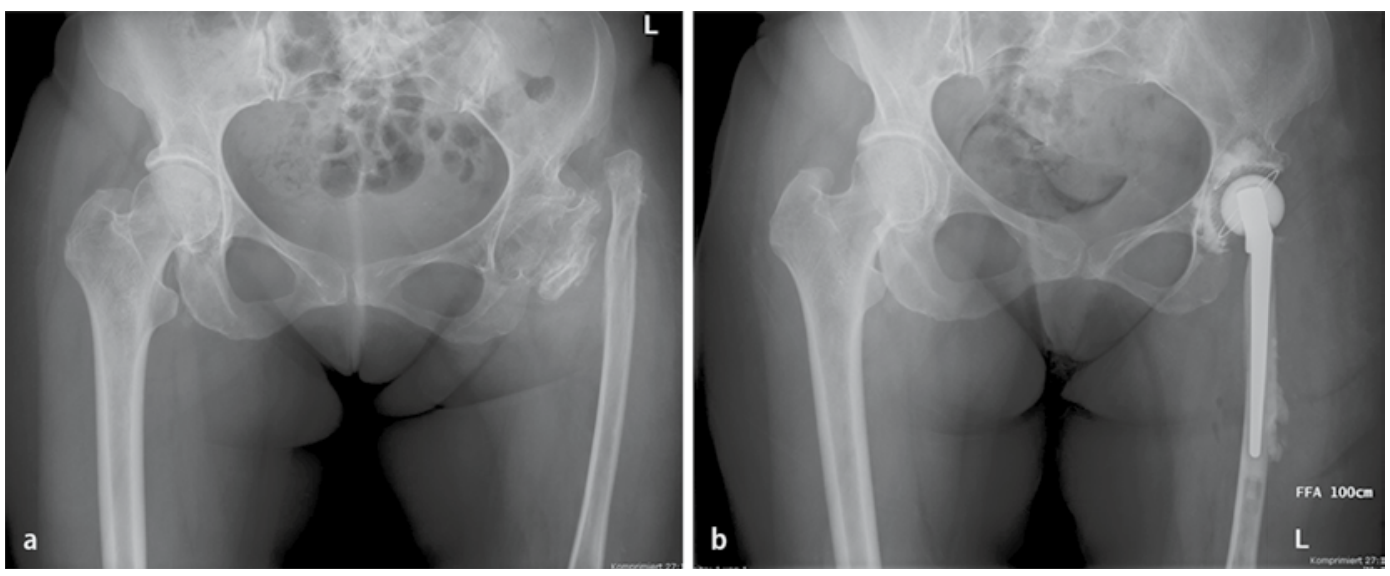

struktion unter Verwendung eines Spezialschaftes gefasst. Hierbei konnte eine akute Verlängerung von $6 \mathrm{~cm}$ realisiert werden (ब Abb. 7b).

\section{Desarthrodese}

Wie oben ausgeführt, entwickeln Patienten längere Zeit nach Hüftarthrodese aufgrund chronisch rezidivierender Überlastungen meist sekundäre degenerative Veränderungen der Lendenwirbelsäule, des kontralateralen Hüftgelenks und beider Kniegelenke. Diese Veränderungen können zu derart ausgeprägten Schmerzen führen, dass die Remobilisierung des versteiften Hüftgelenks wünschenswert wird. Eine weitere Indikation zur Desarthrodese ist die Implantation einer Knietotalendoprothese auf der betroffenen Seite.

Voraussetzung ist zum einen eine adäquate Knochenqualität zur Verankerung von Endoprothesenpfanne und schaft, zum anderen auch der Nachweis, bevorzugt durch MRT und Elektrophy- siologie, noch vorhandener aktivierbarer und funktioneller Reste der pelvitrochanteren Muskulatur. Ein definitiver Verlust der hüftstabilisierenden Muskeln spricht ebenso gegen eine Remobilisierung einer Hüftarthrodese wie ein latent erhöhtes Infektrisiko. Als operationstechnisch ungünstig erwiesen sich auch Arthrodesen, die über einen lateralen Zugang durchgeführt worden waren [9].

Die Rate an operativen Komplikationen ist bei Desarthrodesen erhöht. Tiefe und oberflächliche Infektionen, periprothetische Frakturen, Ischiasläsionen sowie heterotope Ossifikationen mit erneuten Ankylosen wurden gehäuft beschrieben.

Die postoperativen Ergebnisse zeigen bei der überwiegenden Zahl der operierten Patienten funktionelle Einschränkungen mit deutlichem Hinken. Perioperative Komplikationen und eingeschränkte funktionelle Ergebnisse müssen in die Indikation mit einfließen und sind dem Patienten präoperativ ausführlich darzulegen [6]. Der funktionelle Ge- winn ist erwartungsgemäß bei den Patienten am höchsten, deren knöcherne Geometrie nach Hüftarthrodese nicht befriedigt, wie bei dem in $\mathbf{A b b .} 8$ dargestellten Fall: Bei dem jetzt 64-jährigen Patienten war nach posttraumatischer Hüftkopfnekrose links im 14. Lebensjahr eine Hüftarthrodese, seinerzeit durch Ruhigstellung im Becken-Bein-Gips-Verband vorgenommen worden, woraus eine knöcherne Geometrie mit $30^{\circ}$ Flexion, $7 \mathrm{~cm}$ Beinverkürzung links und $20^{\circ}$ Außentorsionsabweichung resultierte (๑ Abb. 8a). Durch eine reorientierende Desarthrodese mit Implantation einer zementierten Hüft-TEP (• Abb. 8b) konnten eine deutliche funktionelle Verbesserung und Linderung der lumbosakralen Schmerzen erreicht werden.

\section{Alternative Operationsverfahren}

Verschiedene Konstellationen nach Destruktion eines Hüftgelenks lassen keines der oben genannten rekonstruktiven Verfahren zu. Daher sind auch heute noch 


\section{Arthrodese vs. Gelenkersatz}
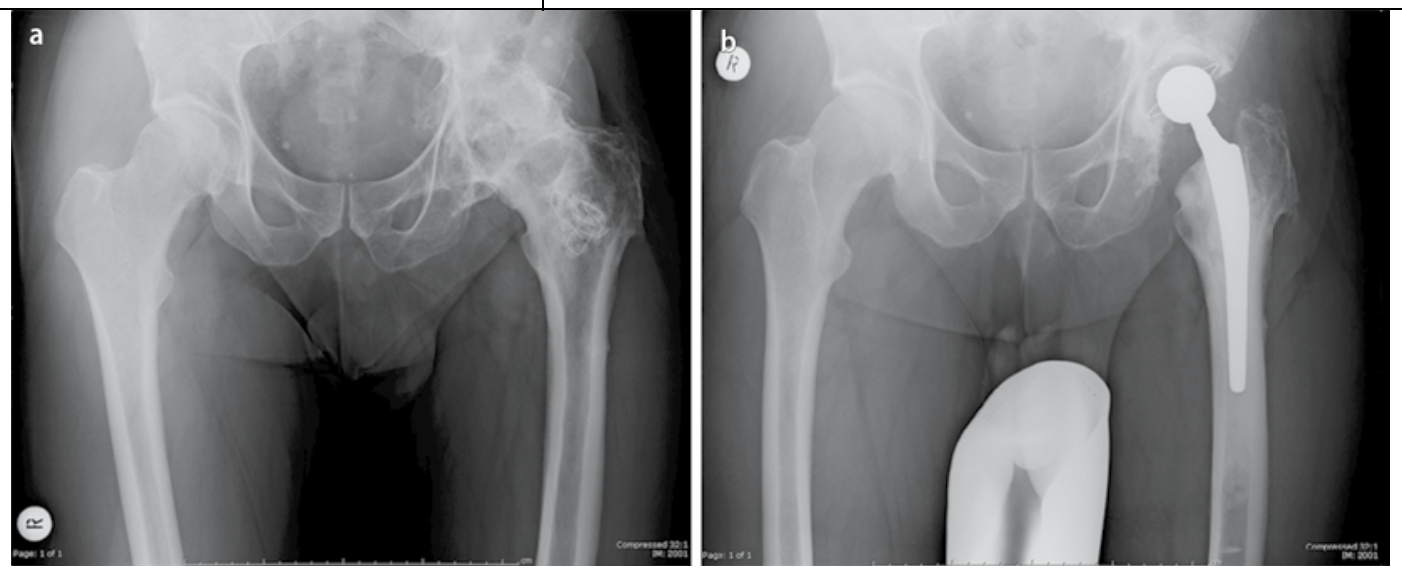

Abb. 8 464-jähriger Patient, a knöcherne Geometrie mit $30^{\circ}$ Flexion, $7 \mathrm{~cm}$ Beinverkürzung links und $20^{\circ}$ Außentorsionsabweichung nach Hüftarthrodese im 14. Lebensjahr, b deutliche funktionelle Verbesserung und Linderung der lumbosakralen Schmerzen nach reorientierender Desarthrodese mit Implantation einer zementierten Hüft-TEP
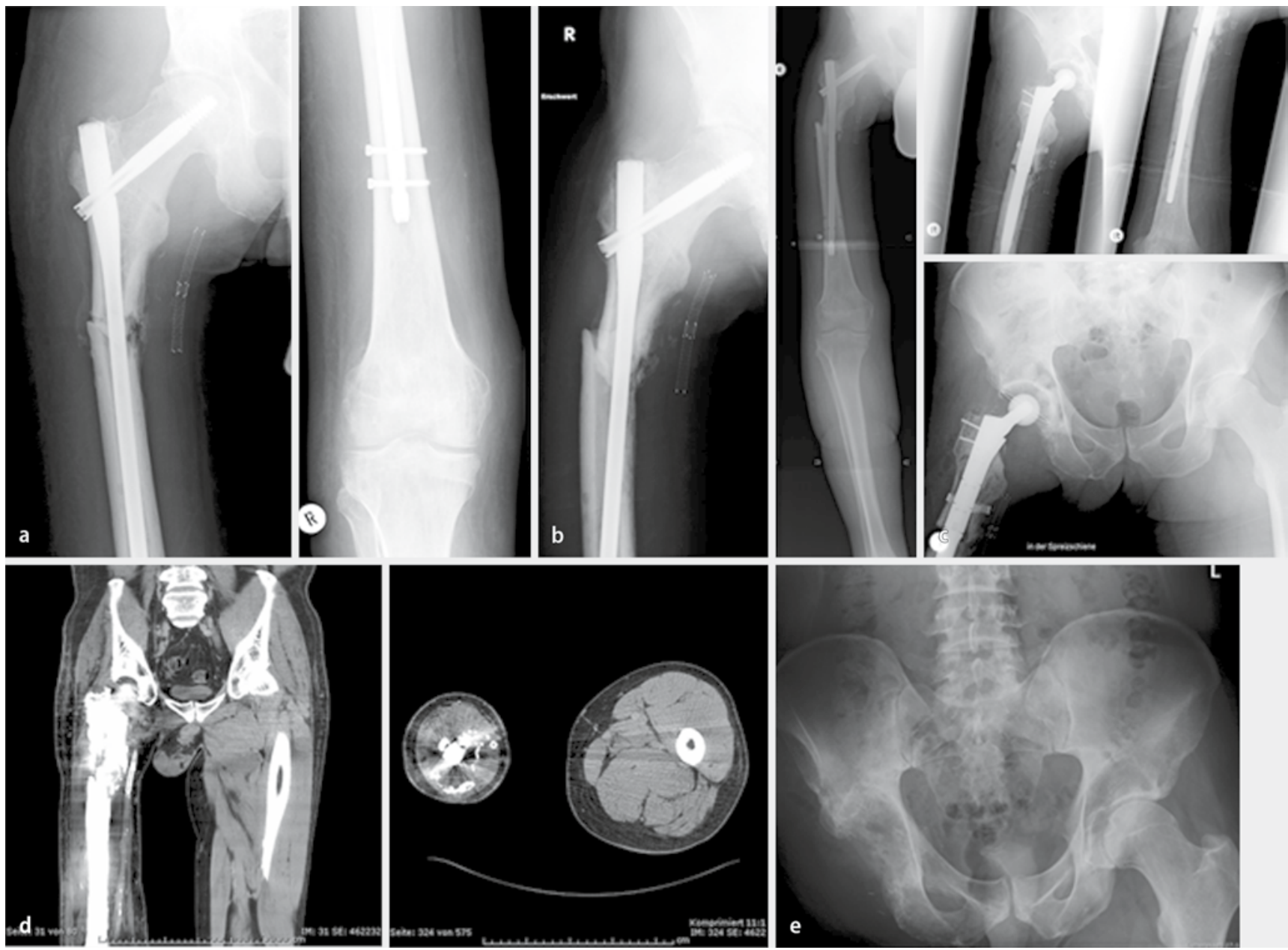

Abb. $9 \Delta$ 40-jähriger Patient mit pathologischer proximaler Femurfraktur rechts, a primäre Stabilisierung der Fraktur durch Marknagelosteosynthese, $\mathbf{b}$ ausbleibende Knochenheilung mit konsekutiver Deformität in der Frakturzone, $\mathbf{c}$ Verfahrenswechsel auf TEP mit Reorientierung der Frakturzone über langen Revisionsschaft, $\mathbf{d}$ tiefer TEP-Infekt rechts, e Hüftexartikulation mit radikaler Revision

resezierende sowie ablatierende Operationen notwendigerweise dem therapeutischen Spektrum der Hüftgelenkchirurgie zuzurechnen.

\section{Girdlestone-Anlage}

Nicht beherrschbare Infektionen nach primärer Endoprothetik oder entsprechenden Wechseloperationen, insbesondere bei immunkompromittierten Patienten oder bei Patienten mit allgemein eingeschränkter Operabilität, indizieren die Resektionsarthroplastik des Hüftgelenks als sinnvolle temporäre oder auch permanente Maßnahme. Die temporäre Girdlestone-Anlage ist notwendiger Bestandteil eines mehrzeitigen Hüftendoprothesenwechsels bei Infektsituationen, 

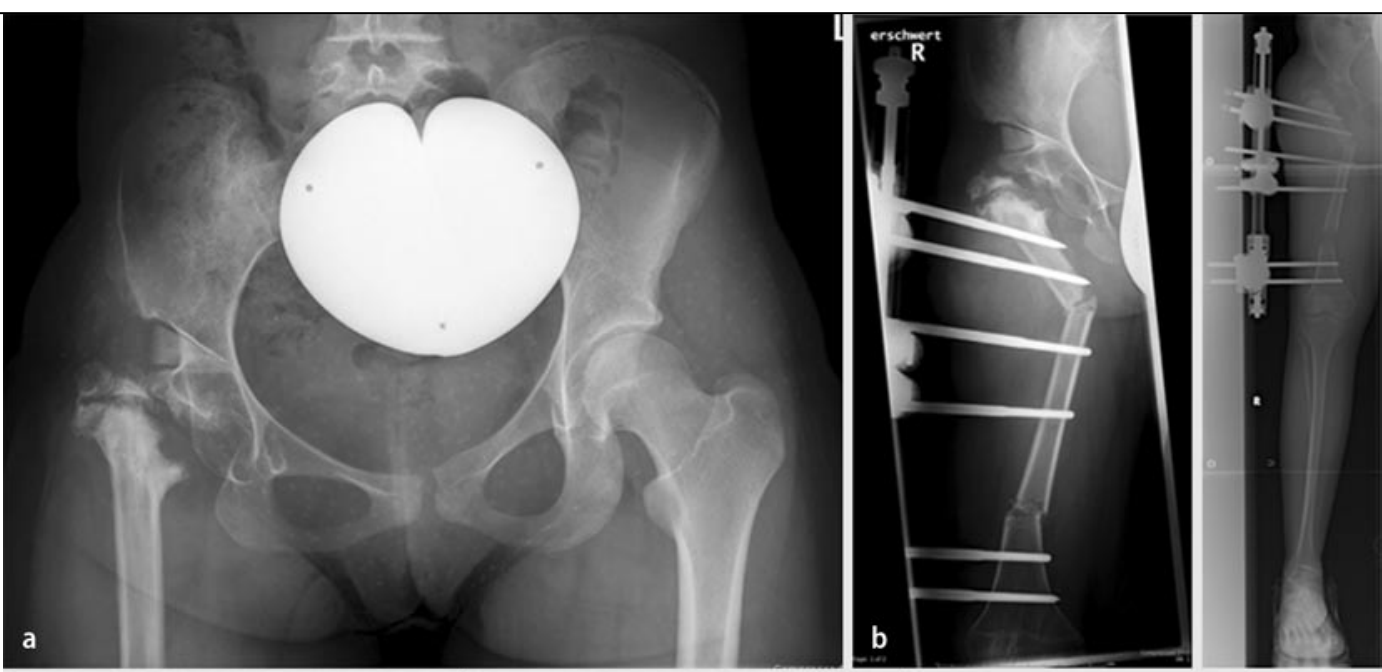

Abb. 10 $>15$-jährige Patientin mit funktioneller und anatomischer GirdlestoneSituation rechts (a), minimalinvasive Bohrloch-MeiBel-Osteoklasie am Übergang vom proximalen zum mittleren Femurviertel mit akuter Valgisation und Extension sowie analoge Osteoklasie am Übergang vom 3. zum 4. Viertel zur anschließenden kontinuierlichen Varisation und Verlängerung (b), Entfernung der externen Fixation nach knöcherner Überbrückung (c)

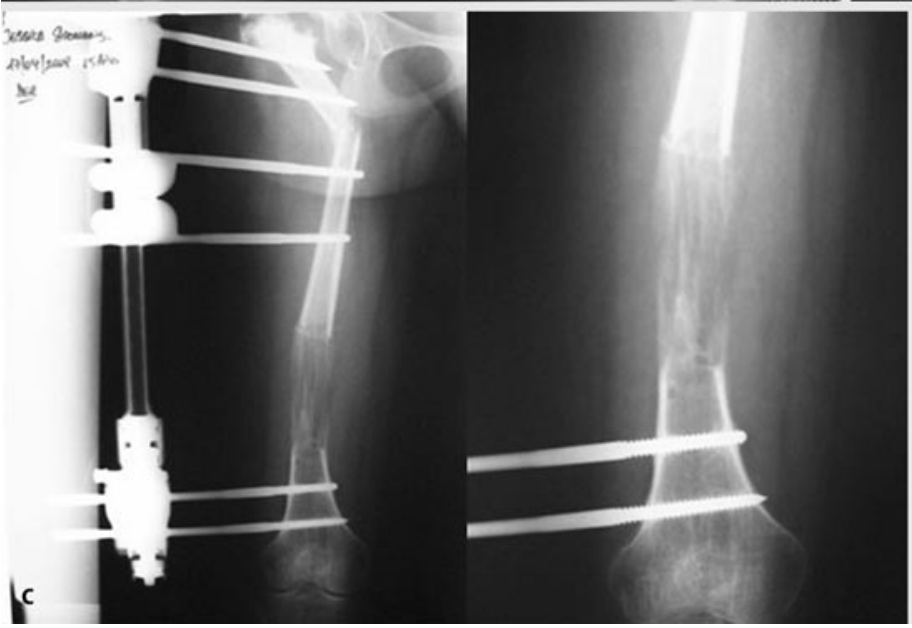

sei es mit oder ohne interponierten Platzhalter aus antibiotikahaltigem Zement oder Ähnlichem.

Über die Indikation und den Zeitpunkt einer alloarthroplastischen Rekonstruktion entscheiden neben dem Allgemeinzustand des Patienten der klinische Verlauf sowie die systemischen und lokalen Infektparameter.

\section{Hüftexartikulation}

Bei nicht beherrschbarerer Problematik im betroffenen Hüftgelenk und/oder völligem Funktionsverlust der ipsilateralen unteren Extremität kann im Einzelfall eine Hüftexartikulation erlösend sein und zur Sanierung der vorbestehenden Situation führen.

In - Abb. 9 ist der Fall eines heute 40 -jährigen Patienten mit pathologischer proximaler Femurfraktur rechts 12 Jahre nach lokaler Resektion eines embryonalen Rhabdomyosarkoms und lokaler Radiochemotherapie und dadurch verursachter hochgradiger Fibrose aller Weichteile einschließlich der Blut- und Lymphgefäße mit Indikation zur Dilatation und Stenting der A. femoralis superficialis dargestellt. Die pathologische Femurfraktur wurde primär durch Marknagelosteosynthese stabilisiert ( $\bullet$ Abb. 9a). Aufgrund der radiogenen Osteonekrose blieb eine Knochenheilung aus, mit konsekutiver Deformität in der Frakturzone (- Abb. 9b). Daraufhin erfolgte der Verfahrenswechsel auf Totalendoprothese mit Reorientierung der Frakturzone über einen langen Revisionsschaft ( $\mathbf{D}$ Abb.9c). 3 Jahre später wurde ein tiefer TEP-Infekt rechts diagnostiziert, der möglicherweise über eine Paronychie im Bereich des rechten asensiblen Fußes ausgelöst worden war. Bei ausgeprägt funktionellem und sensomotorischem Defizit des rechten Beines, der computertomographisch gut ablesbar war (- Abb.9d), erfolgte nach ausgiebiger Abwägung und auf ausdrücklichen Wunsch des Patienten die Hüftexartikulation mit radikaler Revision entsprechend den Regeln der septischen Chirurgie (• Abb. 9e). Postoperativ kam es zur primären Heilung der Weichteile. Nach adäquater prothetischer Versorgung resultierte eine erstaunlich gute Funktion mit einem letztlich hochzufriedenen $\mathrm{Pa}$ tienten.

\section{„Pelvic support osteotomy"}

Typische Spätfolgen einer frühkindlichen bakteriellen Koxitis sind das Fehlen von Hüftkopf und Schenkelhals, ein proximales Hochwandern des Oberschenkels, eine Verkürzung und Hypotrophie des betroffenen Beines, ein gestörtes Gangbild und Schmerzen.

Für diese Problematik eignen sich weder der alloarthroplastische Hüftgelenkersatz noch eine Hüftarthrodese. Hier empfiehlt sich, als Weiterentwicklung der Schanz-Osteotomie, die Hüftrekonstruktion nach Ilisarov. Diese schließt eine akute Valgisations-Extensions-Osteotomie im proximalen Drittel des Femurs 


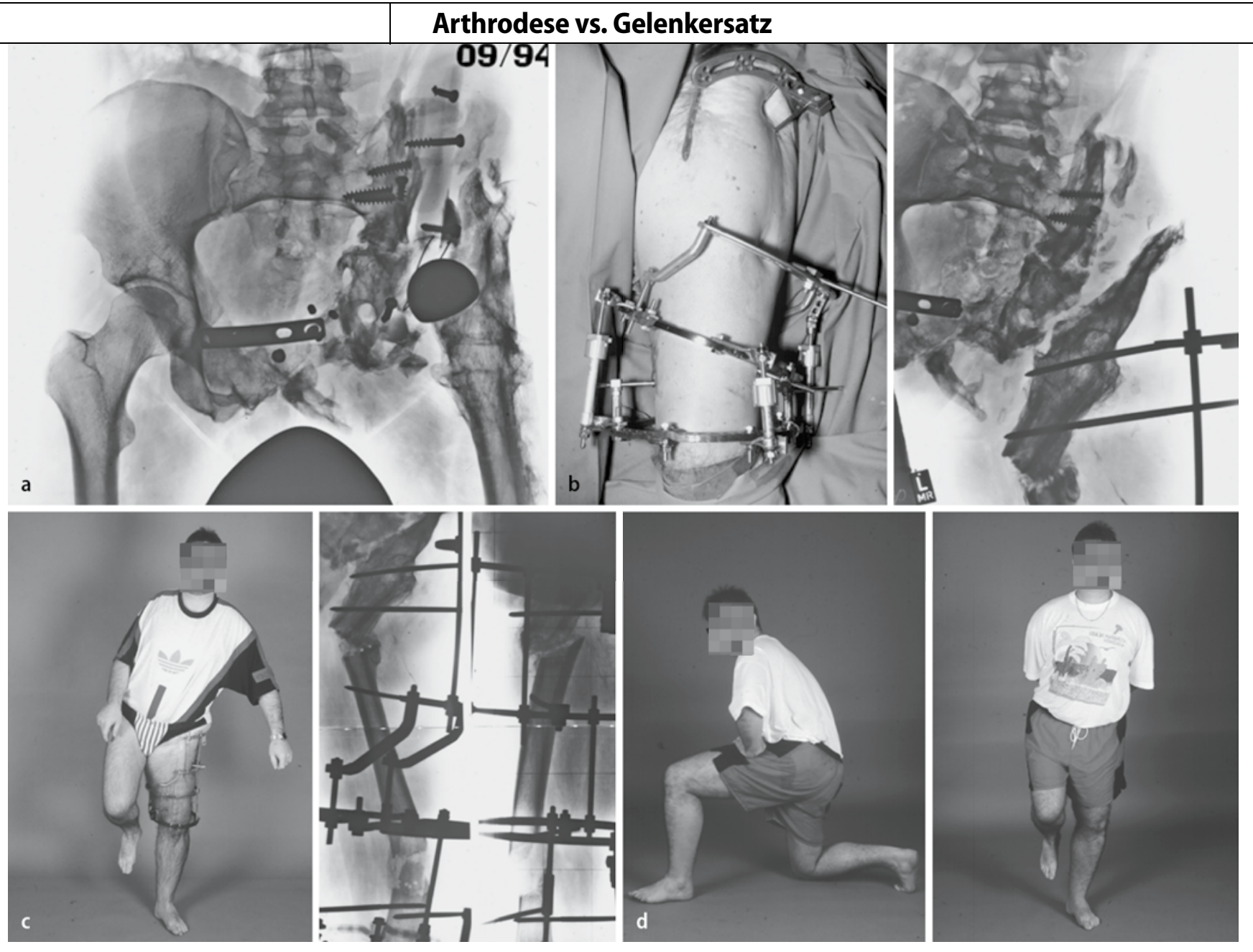

Abb. $11 \Delta$ Zustand nach Hemipelvektomie links bei malignem fibrösem Histiozytom, Defektüberbrückung durch Polyacetalbeckenteilersatz mit gleichzeitiger Implantation einer zementierten Polyethylenpfanne und Überkronung des Hüftkopfes durch eine Wagner-Kappe (a), akute subtrochantere Valgisations-Extensions-Osteotomie (b), Osteoklasie der distalen Femurdiaphyse zur kontinuierlichen Varisation und Verlängerung über llisarov-Ringfixateur (c), sehr gutes funktionelles Ergebnis 1 Jahr nach Fixateurentfernung (d)

ein, in Kombination mit einer kontinuierlichen Varisation und Verlängerung durch eine distale Femurosteotomie [5].

Die funktionellen Ergebnisse sind überraschend gut, wie die Fallbeispiele in - Abb. 10 und 11 zeigen: Bei der in - Abb. 10 vorgestellten 15 -jährigen Patientin lag nach Koxitis während der ersten Lebenstage, inadäquater chirurgischer Therapie in der Frühphase sowie frustraner Azetabuloplastik mit supraazetabulärer Einbringung eines Knochensubstituts eine funktionelle und anatomische Girdlestone-Situation rechts $\operatorname{vor}(-$ Abb. 10a). Neben einer hochgradig schmerzhaften Funktionseinschränkung im rechten Hüftgelenk resultierte eine Verkürzung des rechten Beins um $11 \mathrm{~cm}$. Es wurden eine minimalinvasive Bohrloch-Meißel-Osteoklasie am Übergang vom proximalen zum mittleren Fe- murviertel mit akuter Valgisation und Extension sowie eine analoge Osteoklasie am Übergang vom 3. zum 4. Viertel zur anschließenden kontinuierlichen Varisation und Verlängerung mittels Kallusdistraktion durch unilateralen Distraktionsfixateur vorgenommen (• Abb. 10b). Nach knöcherner Überbrückung proximal im Bereich der Akutkorrektur und distal im Bereich der kontinuierlichen Kallusdistraktion wurde die externe Fixation entfernt (• Abb. 10c).

In Abb. 11 ist der Fall eines 33-jährigen Patienten mit Zustand nach Hemipelvektomie links bei malignem fibrösem Histiozytom vorgestellt. Der Defekt war durch einen proximal am Os sacrum und distal an der kontralateralen Symphyse verankerten Polyacetalbeckenteilersatz überbrückt worden. Gleichzeitig waren eine zementierte Polyethylenpfanne implantiert und der Hüftkopf durch eine Wagner-Kappe überkront worden. Daraus resultierte eine mechanisch hoch instabile und schmerzhafte Situation bei gleichzeitig erheblicher Bewegungseinschränkung (- Abb. 11a). Nach weitgehender Entfernung der erreichbaren Implantate erfolgte eine akute subtrochantere Valgisations-Extensions-Osteotomie ( Abb. 11b) mit anschließender Osteoklasie der distalen Femurdiaphyse zur kontinuierlichen Varisation und Verlängerung über einen kombinierten Ilisarov-Ringfixateur (- Abb. 11c). Aus - Abb. 11d ist das sehr gute funktionelle Ergebnis 1 Jahr nach Entfernung des Fixateur externe ersichtlich.

Nach knöcherner Ausreifung und adäquatem Trainingszustand der pelvi-trochanteren Muskulatur kann grundsätzlich später eine Konversion - nach voran- 
gehender reorientierender Korrekturosteotomie - in einen sekundären alloarthroplastischen Gelenkersatz erwogen werden. Dies dürfte allerdings eher seltenen Ausnahmefällen vorbehalten sein.

\section{Fazit}

Für das destruierte Hüftgelenk lassen sich nur noch sehr ausgewählte Indikationen zur Arthrodese in ausgesuchten Einzelfällen ableiten. Dies kann im Einzelfall bei jüngeren Männern mit überdurchschnittlicher körperlicher Belastung und gleichzeitig adäquater Qualität von Weichteilen und Knochen erwogen werden. Langfristig ist die Versteifung des Hüftgelenks, selbst in allgemein anerkannten geometrischen Dimensionen, funktionell ungünstig und wirkt sich dementsprechend negativ auf die benachbarten Gelenke aus, namentlich auf die Lendenwirbelsäule, auf das kontralaterale Hüftgelenk und auf beide Kniegelenke. Die Indikation zur Hüftarthrodese wird nicht nur durch die enormen Fortschritte in der rekonstruktiven Hüftalloarthroplastik eingeschränkt, sondern darüber hinaus durch konkurrierende, funktionell durchaus überzeugende Operationstechniken, wie etwa die „pelvic support osteotomy". Letztlich bleibt die Differenzialtherapie immer eine Einzelfallentscheidung und muss der individuellen anatomischen und funktionellen Situation ebenso Rechnung tragen wie der Persönlichkeit und der Kooperationsbereitschaft des betroffenen Patienten.

\section{Korrespondenzadresse}

\section{Prof. Dr. W. Strecker}

Klinik für Orthopädie und Unfallchirurgie,

Klinikum am Bruderwald,

Buger Straße 80, 96049 Bamberg

chirurgie2@sozialstiftung-bamberg.de

Interessenkonflikt. Keine Angaben

\section{Literatur}

1. Callaghan JJ, Brand RA (1985) Hip arthrodesis: a long-term follow-up. J Bone Joint Surg Am 67:1328-1335

2. Joshi AB, Porter ML, Trail IA et al (1993) Long-term results of Charnley low-friction arthroplasty in young patients. J Bone Joint Surg Br 75:616-623
3. Karol LA, Halliday SE, Gourineni P (2000) Gait and function after intra-articular arthrodesis of the hip in adolescents. J Bone Joint Surg Am 82:561-569

4. Nieder E, Engelbrecht E, Steinbrink K et al (1990) The saddle prosthesis for salvage of the destroyed acetabulum. J Bone Joint Surg Br 72:1014-1019

5. Rozbruch RS, Paley D, Bhave A, Herzenberg JE (2005) Ilizarov hip reconstruction for the late sequelae of infantile hip infection. J Bone Joint Surg Am 87:1007-1018

6. Schuh A, Zeiler G, Werber S (2005) Ergebnisse und Erfahrungen der Remobilisation von Hüftarthrodesen. Orthopäde 34:218-224

7. Siebenrock K, Gautier E, Matta J et al (1996) Neue Technik einer Hüftarthrodese mit einem anterioren Zugang und ventraler Platte. Orthopäde 25:140-145

8. Sofue M, Kono S, Kawaji W, Homma M (1989) Long term results of arthrodesis for severe osteoarthritis of the hip in young adults. Int Orthop 13:129-133

9. Wagner M, Wagner H (1996) Die Arthrodese des Hüftgelenkes mit der Kreuzplatte. Indikation, Technik, Ergebnisse. Orthopäde 25:129-139

10. Zeiler G, Schuh A (2004) Die Arthrodese des Hüftgelenkes und ihre Remobilisation. Orthopäde 33:939-956 